\title{
UPPER GASTROINTESTINAL VISCERAL PAIN MODELS IN GUINEA-PIGS (MINI-REVIEW)
}

\author{
Halicka J., Tatar M.
}

Department of Pathological Physiology, Jessenius Faculty of Medicine in Martin, Comenius University, Slovak Republic

\begin{abstract}
Visceral pain brings deep medical burden to society, and it is clinically often the first symptom which leads to a medical consultation. In the time of deeper understanding of molecular transducers in pain pathways, there is still need for models to test hypothesis how would selective inhibition of nociceptive pathways modulates the behavior of experimental animal. In comparison with somatic pain, there is less visceral pain models, probably due to complex extrinsic (dual innervation by dorsal root ganglia and vagus nerve innervation) and intrinsic innervation. We want to develop a new model of visceral pain in guinea pigs to test our hypothesis that vagal afferent innervation affects perception of visceral pain. In this mini-review we would like to closely describe advantages and drawbacks of visceral pain models used in our department.
\end{abstract}

Keywords: guinea pig, visceral pain, pain model, vagus nerve,

\section{INTRODUCTION}

Chronic visceral pain is clinically one of the most common reasons for visits to general practicioner's office [1]. Although essential for host survival, pain can become unbearable especially when chronic. Unexplained abdominal pain represents a significant healthcare burden and one in which there is a current lack of effective treatments. Despite an enormous effort, an integrated understanding of the pathophysiologic mechanisms of chronic visceral pain, particularly with respect to functional gastrointestinal disorders, remains incomplete. A key to discovering novel therapeutic approaches inevitably involves understanding of basic pathophysiology of visceral pain perception. Currently our knowledge about pain perception in animal models is limited and majority of what is known about pain and nociceptors originates from studies of somatic structures (non-visceral components of the body, principally skin). Nevertheless, the most common pain produced by disease and the most difficult to manage is that originating from the internal organs [2]. This review is denoted to study nocifensive responses in guinea-pig model of acute and chronic visceral pain from upper gastrointestinal tract.

\section{Guinea pigs in pain studies}

Guinea pigs are widely used in studies of vagal afferent innervation of gastrointestinal tract as described earlier in work of Kollarik and col. [8,9]. Guinea pig's esophagus is a suitable model to study the visceral pain for following reasons: a) guinea pig's vagal afferent nerves are most similar to human from all laboratory animals other than primates, which are unavailable (example: pharmacological properties of respiratory vagal mediated reflexes are in humans and guinea pigs almost identical, which is not relevant for rats and mice), b) esophagus is simpliest visceral organ for studying vagal afferent nerves, c) existence of verified molecular tools for selective inhibition of vagal afferent innervation in guinea pig (adeno-associated virus vectors with shRNA Navl.7 used in [15]).

Address for correspondence:

Juraj Halicka, Department of Pathophysiology, JFM CU, Sklabinska 26, 03601 Martin, Slovakia; e-mail: euraay@gmail.com] 


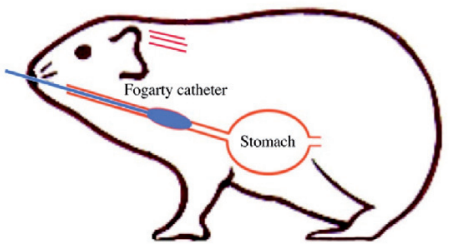

Fig. 1 Acute pain model - noxious acute mechanical distention of the esophagus with naso-esophageal Fogarty catheter insertion. EMG is taken from 3 electrodes inserted into $\mathrm{m}$. acromiotrapezius.

\section{Vagus nerve role in visceral pain}

Innervation of visceral organs differs considerably from innervation of somatic structures. Main difference is dual afferent innervation. Afferent nerves are provided from dorsal root ganglia and vagal ganglia, respectively. It is known that vagus nerve innervates the vast majority of internal organs and almost $80 \%$ of the vagal fibers are afferent, but until recently they were attributed only to a role in reflex circuits $[3,4,5]$. Recent discoveries have shown that patients with high spinal cord injury feel the pain caused by esophageal noxious distension [6] and gastric noxious distension [7]. Former Kollarik's and col. [8,9] work shows population of afferent vagal nerves in guinea pigs esophagus capable of sensing and encoding chemical (acid, adenosin, capsaicin, bradykinin) and mechanical noxious (mechanical distension $>60 \mathrm{mmHg}$ ) signals $[8,9]$.

There are existing rat models of visceromotoric response (VMR) to noxious distension of gastrointestinal system. Ozaki and col. [12] found no change in VMR as a response to gastric distention after vagotomy. Lamb and col. [13] found vagal afferent nerves from stomach sensitive to chemical nociception but not dorsal root ganglia (DRG) afferent nerves. Sakurai and col. [11] interprets data that vagus nerve resection do not affect VMR to gastric distention, but we can also see only partial effect of splanchnectomy (DRG ressection), and we can anticipate that combination of vagotomy/splanchnectomy would be of higher efficiency. Furuta and col. [10] findings of chronic pain model in rats suggest that subdiaphragmatic vagal dysfunction cause chronic muscle hyperalgesia accompanied by visceral pain. Vagal afferent participation on visceral nociception is therefore not clear in literature. While the contribution of the vagus nerve to visceral pain remains unresolved, the vagus nerve clearly plays an important role in the prominent autonomic and emotional reactions to visceral stimuli [14]. Therefore, new models of visceral pain in guinea pigs are necessary to describe function of vagal afferent innervation in perception of visceral pain.

\section{Objective assessment of the pain behaviour in animal models}

In 1979, the International Association for the Study of Pain (IASP) defined pain as "an unpleasant sensory and emotional experience associated with actual or potential tissue damage or described in terms of such damage". With respect to scientific opportunities, animal models of pain often exhibits limitations when it comes to modeling of the complex processes unique to human functions. Never the less many models of visceral pain described in the literature focus primarily on acute visceral nerve activation using mechanical distension, chemical irritation, and/or inflammation [16]. To name few, techniques that are used to measure the visceral pain: viscero-motoric response [17], voluntary movements [18], vocalisation [19], c-fos expression [20], functional MRI [21], grimace scales [22], production 
of stress hormones and cardiovascular reflexes. There is scarce evidence of true chronic pain models, which are in our opinion, affected less by limitations of pain modeling in animals. In our department we are currently using the VMR analysis in acute pain model and voluntary movement analysis in a chronic pain model.

\section{Guinea pig model of acute visceral pain}

Many of the behaviors that are thought to represent spontaneous pain have been reported in different animal models of persistent, inflammatory and neuropathic pain to measure the nature of animal pain experience [2]. Measurement of acute visceral pain in animals is in current model based on brain stem VMR [14]. Viscero-motor reflexes, which were first described by Sherrington in decerebrated animals, is an unconscious respose to noxious stimuli, without consciousness playing a role in pain processing [26]. It is possible to use it as an objective measure of response to painful stimuli. Data are obtained from electromyography (EMG), take advantage of one aspect of behavioral responses to noxious stimuli that are present in many species, including humans, which cause twitching/higher muscle tonus of acromiotrapezius muscle in guinea pig. As the noxious stimulus mechanical noxious distension of esophagus mediated via naso-esophageal Fogarty catheter was used.

Electromyography is a more objective measure of nociceptive behavior than observational techniques [17]. The principal advantage of EMG compared to observation to quantify behavior is that the former provides a direct measure of the electrical activity generated within a specific muscle group during contraction, and is, therefore, not inherently influenced by experimenter bias. Advantage of this technique is the long-term stability of the EMG electrodes which permits chronic time-course studies in addition to acute interventions. Major drawback is a nature of muscle electrical activity, which means animals must be calm and without any movement prior to EMG recording. Each movement is therefore, affecting EMG recordings and reducing validity and possibility to analyze data correctly.

Discussion about usefulness of acute pain models that are measuring response to severe, acutely induced pain, e.g. model of acute VMR of esophageal distension [13,23], is under debate worldwide. To date, there has been limited animal research evaluating the kind of dull, diffuse and persistent visceral pain in animals frequently observed clinically in humans. For addition, even if one model is called chronic visceral inflammatory pain [14], the actual nocifensive behaviour, visceromotor change, vocalization or increase in c-fos expression is caused by acute stimuli (personal discussion with Prof. Peter Holzer, Medical University Graz, Austria).

\section{Guinea-pig model of chronic visceral pain}

The evaluation of chronic pain symptoms and the interpretation of their objective findings in experimental models is problematic. Major problem is that pain symptoms are only nocifensive responses to noxious stimuli. Stressfull conditions during experiment strongly affect nocifensive response in awake animals. However, prolonged experiment with telemetric system brings advantage of significantly lower stress induced by the observer - experimental animal relationship. We hypothesized that chronic visceral pain model could bring more complex nocifensive response evaluation in comparison with acute model.

Miwa et col. [18] describe rat's model of reflux esophagitis, where changes in voluntary movement of esophagitis model reflects visceral symptoms and serve as an index of chronic abdominal symptoms. On the basis of their experiment we set up simple experimental model of guinea pig with reflux esophagitis. Chronic gastroduodenal reflux model, is induced by postpyloric duodenal narrowing and pneumatic dilation of lower esophageal sphincter (Fig. 2). New telemetric state of the art system (Fig. 2; Data Science International, MN, USA) is designed for longterm sampling (up to 6 weeks, depends on implanted transmitter) of any biopotential (electroencephalography, electrocardiography, electromyography, etc.), body temperature, blood pressure, nerve activity and locomotor activity with video recording of each animal in separate cage (example in fig. 3). We use transmitters with one biopoten- 


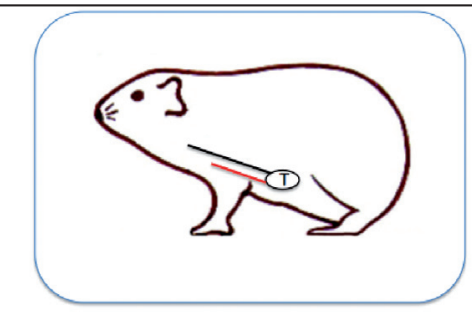

\section{Recelver}

Fig. 2. Scheme of telemetric system - animal, implanted with transmitter (T) with 2 electrodes leads for 1 biopotential acquisition (e.g. ECG). Each animal is housed alone in plastic cage and put upon single receiver.

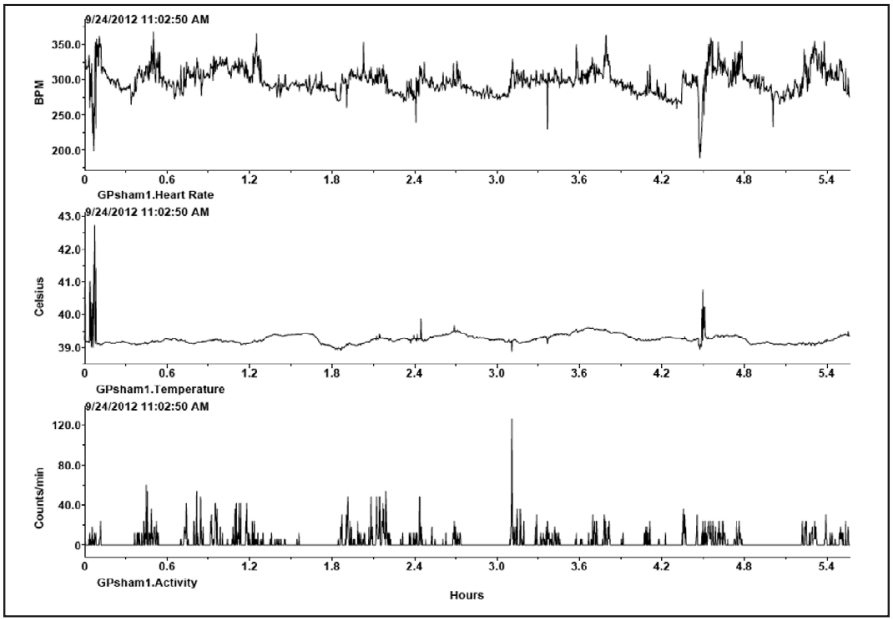

Fig. 35.5 hours (x axis) example of recording from telemetric system of ECG (in BPM on y axis, $1^{\text {st }}$ line), body temperature (in degrees of Celsius on y axis, $2^{\text {nd }}$ line) and animal locomotor activity (in counts/minute on y axis, $3^{\text {rd }}$ line).

tial recording possibility (electrocardiography, ECG). ECG data allow to measure heart-rate variability as viscero-vegetative reflex and brings another objective finding for this experimental model. 
Different anatomy of the upper GIT in guinea pigs is a major drawback of model of chronic visceral discomfort currently in use. Guinea pig's stomach is a so-called stuffed stomach with very few muscles and thin stomach wall. Therefore it is not able to transport the chewed food to the next parts of the digestive system without help. This task is taken over by the next meal. Consequently the appropriate feed for this animal must be low in calories and continuos. Therefore, guinea pigs are prone to gastric dilation volvulus, if used duodenal obstruction would be very narrow.

\section{CONCLUSSION}

Visceral gastrointestinal pain and functional gastrointestinal disorders have attracted increasing attention in recent years [25]. The mechanisms are complicated and poorly understood. The first step for better understanding of the visceral pain would be the development and validation of an objective evaluation method, which allows analysis of symptoms in experimental models of visceral pain. Vagal afferent participation on visceral nociception is therefore not clear in literature.

We are currently working on two different models of visceral pain. Current acute visceral pain models got limitations of presenting visceral pain main characteristics - dull, diffuse and persistent pain - main reason for general practicioner's office visit by majority of patients. To this date more reasonable seems to be a model of chronic visceral pain which is capable of long-term distress observation of animals with chronic visceral pain. This work would like to show better way for development of new models of visceral pain with more physiological setup in the future.

\section{REFERENCES}

1. Christianson JA, Bielefeldt K, Altier C, Cenac N, Davis BM, Gebhart GF, High KW, Kollarik M, Randich A, Undem B, Vergnolle N. Development, plasticity and modulation of visceral afferents. Brain Res Rev. 2009;60(1):171-86.

2. Gold MS, Gebhart GF. Nociceptor sensitization in pain pathogenesis. Nat Med. 2010 Nov;16(11):1248-57.

3. Berthoud HR, Neuhuber WL. Functional and chemical anatomy of the afferent vagal system. Auton Neurosci. 2000 Dec 20;85(1-3): 1-17

4. Cervero F. Sensory Innervation of the Viscera: Peripheral Basis of Visceral Pain. Physiol Rev. 1994 Jan;74(1):95-138

5. Ness TJ, Gebhart GF. Visceral pain: a review experimental studies. Pain. 1990 May;41(2):167-234.

6. DeVault KR, Beacham S, Castell DO, Streletz LJ, Ditunno JF. Esophageal sensation in spinal cord-injured patients: balloon distension and cerebral evoked potential recording. Am J Physiol. 1996 Dec;271(6 Pt 1):G937-41.

7. Levinthal DJ, Bielefeldt K. Pain without nociception? Eur J Gastroenterol Hepatol. 2012 Mar;24(3):336-9.

8. Yu S, Undem BJ, Kollarik M. Vagal afferent nerves with nociceptive properties in guinea- pig oesophagus. J Physiol 2005;563(Pt 3):831-42.

9. Kollarik M, Ru F, Undem BJ. Acid-sensitive vagal sensory pathways and cough. Pulm Pharmacol Ther 2007; 20: 402-411.

10. Furuta S, Shimizu T, Narita M, Matsumoto K, Kuzumaki N, Horie S, Suzuki T, Narita M. Subdiaphragmatic vagotomy promotes nociceptive sensitivity of deep tissue in rats. Neuroscience. 2009 Dec 15;164(3):1252-62.

11. Sakurai J et al.. Activation of Extracellular Signal-Regulated Protein Kinase in Sensory Neurons After Noxious Gastric Distention and Its Involvement in Acute Visceral Pain in Rats. Gastroenterology 2008;134:1094-1103.

12. Ozaki N, Bielefeldt K, Sengupta JN, Gebhart GF. Models of gastric hyperalgesia in the rat. Am J Physiol Gastrointest Liver Physiol 283:666-676, 2002.

13. Lamb K, Kang YM, Gebhart GF, Bielefeldt K. Gastric inflammation triggers hypersensitivity to acid in awake rats. Gastroenterology 2003;125:1410-1418.

14. Fioramonti J, Gebhart GF. In vivo and transgenic animal models used to study visceral hypersensitivity. Neurogastroenterol Motil. 2007 Jan;19(1 Suppl):20-8.

15. Kollarik M, Carr MJ, Ru F, Ring CJ, Hart VJ, Murdock P, Myers AC, Muroi Y, Undem BJ. Transgene expression and effective gene silencing in vagal afferent neurons in vivo using recombinant adeno-associated virus vectors. J Physiol. 2010 Nov 1;588(Pt 21):4303-15.

16. Westlund KN. Animal models of visceral pain. In: Chao Ma and Jun-Ming Zhang (eds.), Animal Models of Pain, Neuromethods, vol. 49 Humana Press 1st Edition. edition (October 11, 2010). p 41-68. 
17. Jones RCW. Models of Visceral Pain: Colorectal Distension (CRD). Current Protocols in Pharmacology. Online Posting Date: September, 2004 Sep 1; Chapter 5:Unit 5.36.

18. Miwa H, Oshima T, Sakurai J, Tomita T, Matsumoto T, Iizuka S, Koseki J. Experimental oesophagitis in the rat is associated with decreased voluntary movement. Neurogastroenterol Motil. 2009 Mar;21(3):296-303.

19. Kurejova M, Nattenmuller U, Hildebrandt U, Selvaraj D, Strosser S, Kuner R. An improved behavioural assay demonstrates that ultrasound vocalizations constitute a reliable indicator of chronic cancer pain and neuropathic pain. Mol Pain. 2010 Mar 26;6:18.

20. Dragunow M, Faull R. The use of $\mathrm{c}$-fos as a metabolic marker in neuronal pathway tracing. J Neurosci Methods. 1989 Sep;29(3):261-5.

21. Westlund KN, Vera-Portocarrero LP, Zhang L, Wei J, Quast MJ, Cleeland CS. fMRI of supraspinal areas after morphine and one week pancreatic inflammation in rats. Neuroimage. 2009 Jan 1;44(1):23-34.

22. Keating SC, Thomas AA, Flecknell PA, Leach MC. Evaluation of EMLA cream for preventing pain during tattooing of rabbits: changes in physiological, behavioural and facial expression responses. PLoS One. 2012;7(9):e44437.

23. Banner SE, Sanger GJ. Differences between 5-HT3 receptor antagonists in modulation of visceral hypersensitivity. Br J Pharmacol 1995; 114: 558-62.

24. Nijsen MJ, Ongenae NG, Coulie B, Meulemans AL. Telemetric animal model to evaluate visceral pain in the freely moving rat. Pain. 2003 Sep; 105(1-2):115-23.

25. Haycox A, Einarson T, Eggleston A. The health economic impact of upper gastrointestinal symptoms in the general population: results from the Domestic/International Gastroenterology Surveillance Study (DIGEST). Scand J Gastroenterol Suppl 1999; 231: 38-47.

26. Sherrington SC. The integrative action of the nervous system. New Haven: Yale University Press; 1906.

Received: January,10,2013

Accepted: March, 12, 2013 